\title{
АЛГОРИТМ ВРЕМЕННОЙ КЛАСТЕРИЗАЦИИ УЗЛОВ БЕСПРОВОДНЫХ СЕНСОРНЫХ СЕТЕЙ ДЛЯ СБОРА ИНФОРМАЦИИ МОНИТОРИНГА С ИСПОЛЬЗОВАНИЕМ БПЛА
}

Аннотащия: Предложен алгоритм кластеризации в беспроводных сенсорных сетях (БСС) с помощью беспилотного летательного аппарата (БпЛА), выполняющего роль временного главного узла кластера. Данный алгоритм кластеризации основан на модифицированном алгоритме FOREL, позволяющий минимизировать количество точек съема информации (кластеров) в маршруте облета и оптимизировать зону радиосвязности БпЛА. Предложены правила уменьшения расхода энергии узлов при взаимодействии с БпЛА.

Ключевые слова: беспроводные сенсорные сети, кластеризация, FOREL, сбор информации с БПЛА.

Постановка задания в общем виде. Современные технологии беспроводных сенсорных сетей (БСС) становятся популярными в различных областях применения (наблюдение за лесными массивами, полями, нефтепроводами и газопроводами, границами, осуществление экологического и метеорологического мониторинга, поисково-спасательные миссии и т.п.) в том числе и в отдаленных (недоступных) районах, где отсутствует телекоммуникационная инфраструктура [1 - 9]. БСС может быть предназначена для работы в течение нескольких месяцев или даже лет в труднодоступных районах. Для сбора данных в таких условиях предлагается использовать БПЛА. Автономные сенсорные узлы осуществляют мониторинг определенных параметров зон своего покрытия, хранят полученные данные и ждут возможности их передачи на центр сбора информации через БпЛА при появления его в зоне радиосвязности.

БпЛА облетают интересующую территорию и собирают данные мониторинга от сенсорных узлов. При облете территории по определенному маршруту БпЛА устанавливает радиосвязь с сенсорными узлами, принимает от них данные мониторинга, хранит и по возвращении передает на центр сбора данных для их дальнейшего анализа.

Сенсорные узлы имеют ограниченные ресурсы по: энергии батареи, производительности процессора, памяти, мощности передатчика, пропускной способности радиоканала и т.д. Современные БСС могут состоять из сотен или даже тысяч сенсорных узлов. Замена батарей для такого большого количества узлов может быть непрактичной или даже не возможной. Следовательно, уменышение (перераспределение) расхода энергии сенсорными узлами имеет решающее зна-

(C) А.В. Романюк 
Міжвідомчий науково-технічний збірник «Адаптивні системи автоматичного управління» № 2’ (33) 2018

чение для увеличения времени их функционирования. Одним из способов решения данной задачи является кластеризация сети, позволяющая сократить длину маршрута облета и увеличить время функционирования БСС. Поэтому разработка алгоритмов кластеризации в БСС с использованием БпЛА является актуальной научной задачей.

\section{Анализ последних публикаций и направления решения задачи.}

Процесс кластеризации в БСС зависит от способа сбора данных:

1. Способ рандеву [2 - 5, 7-9].

По определенному алгоритму (централизовано или децентрализовано в зависимости от наличия информации о состоянии сети в центре управления сетью) сеть разбивается на кластеры. Узлы, обменявшись служебными сообщениями, на основе принятых критериев выбирают узлы-рандеву. В каждом кластере строятся маршруты передачи данных от узлов-мониторинга до узлов-рандеву, которые собирают и хранят данные мониторинга узлов своего кластера сети до подлета БПЛА. Далее БпЛА или центр управления сетью строит маршрут облета и сбора данных от узлов-рандеву.

Достоинства: использование узлов-рандеву позволяет снизить энергопотребление узлов кластера, уменьшить время полета и тем самым ускорить время сбора информации мониторинга. Недостатки: для обеспечения способа рандеву необходимо реализовать ряд дополнительных алгоритмов управления сетью, что ведет к дополнительному служебному трафику и расходу энергии батарей. Кроме этого, объемы данных обрабатываемых в узлах-рандеву, особенно в больших сетях, могут быть значительными, что может привести к переполнению буферов и задержкам доставки данных при передаче на БпЛА.

2. Способ непосредственного сбора данных от узлов к БпЛА [1, 2, 6, 9].

Центр управления сетью (или БпЛА), основываясь на информации о координатах узлов, вычисляет точки сбора информации (например, как “центр массы кластера"), формирует кластер на время сбора. Маршрут полета БпЛА формируется из точек сбора данных.

Достоинства: отсутствие дополнительных алгоритмов управления. Недостаток: невозможность децентрализованной реализации.

Выделим основные различия рассмотренных способов. При первом способе - узлы сами формируют реальные связные кластеры и выбирают главный узел (узел-рандеву). При втором - формируются временные кластеры самим БПЛА, выполняющего временно роль главного узла кластера.

При формировании кластеров целевыми функциями управления сети могут быть $[7,8]$ : минимизация времени сбора данных мониторинга, увеличение вре- 
мени функционирования сети $T_{\phi}$ (за счет совершенствования алгоритмов взаимодействия между БпЛА и сенсорными узлами в процессе сбора данных мониторинга), минимизация количества БпЛА и др.

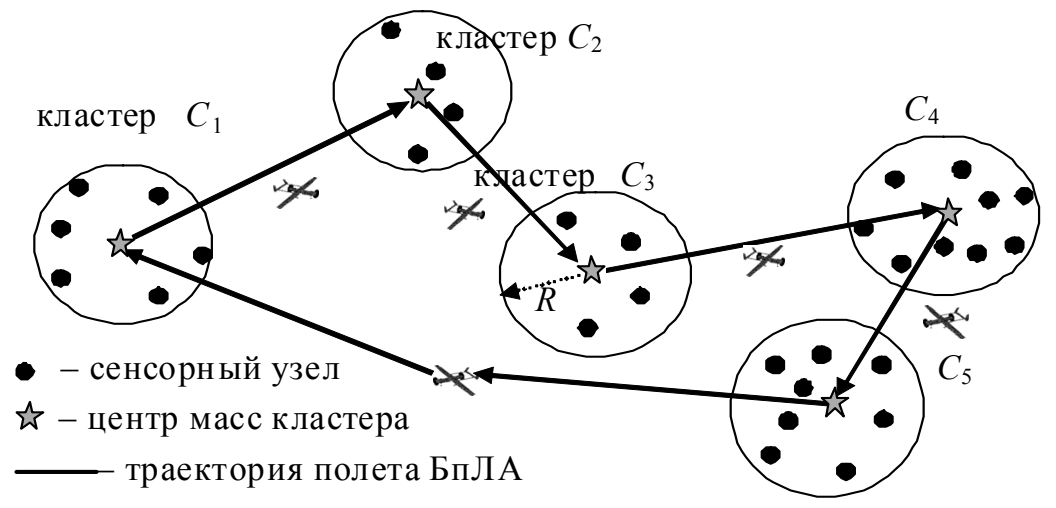

Puc. 1. Вариант перемещения БпЛА между точками сбора - “центрами масс кластеров”

В работах [2, 6, 9] задача кластеризации БСС и сбора данных БпЛА непосредственным способом рассматривается в отрыве от необходимости учета нескольких целевых функций управления.

Цель статьи: разработать алгоритм кластеризации и сбора информации мониторинга БПЛА непосредственно с узлов БСС, позволяющий сократить длину маршрута (и, соответственно, время сбора данных), уменьшить расход энергии батарей сенсорных узлов за счет различных правил сбора данных в полученных кластерах (и, соответственно, увеличить время функционирования сети), минимизировать число используемых БпЛА.

\section{Изложение основного материала.}

Модель сети. Рассматривается неоднородная БСС. Наземные узлы случайным образом распределены на определенной территории, имеют одинаковые функции и ресурсы, стационарны, не обслуживаются, не изменяют своего местоположения, оснащены системой позиционирования (например, GPS). БпЛА и сенсорные узлы оснащены одинаковым радиооборудованием и поддерживают одинаковые протоколы информационного обмена (например, IEEE 802.11), имеют ограниченные дальность радиосвязности и скорость обмена. Каждый узел сети имеет собственную систему управления, действует в кооперации с другими узлами сети и БпЛА, имеет достаточно памяти для хранения данных мониторинга.

БПЛА имеет возможность перемещаться в трех измерениях с переменной скоростью на ограниченной высоте и ограниченное время. БПЛА обладает соб- 
Міжвідомчий науково-технічний збірник «Адаптивні системи автоматичного управління» № 2' (33) 2018

ственной системой управления, позволяющей самостоятельно принимать свои решения в условиях отсутствия связности с центром управления сетью. Когда в зону радиосвязности узла входит БПЛА, он отправляет ему собранные данные мониторинга.

Считается, что БпЛА знает координаты узлов, которые могут быть получены одним из следующих способов:

1. На этапе развертывания наземных сетей при детерминированном размещении узлов - фиксируются координаты каждого из узлов при его размещении.

2. При случайном развертывании узлов сети БпЛА осуществляет первичный облет территории, покрываемой наземными узлами БСС, и собирает данные о координатах узлов в предположении наличия в узлах системы позиционирования. В этом случае маршрут полета строится с целью покрытия всей территории наблюдения. В процессе облета БпЛА собирает как информацию мониторинга, так информацию о состоянии узлов и кластеров для дальнейшего планирования задач управления.

3. При наличии связной топологии БСС и возможности сбора центром управления информации о состоянии и координатах расположении сенсорных узлов.

Будем полагать, что зона радиосвязности БПЛА представляет собой круг радиуса $R$, а считывание информации с узлов БСС может производиться в произвольных точках.

Нахождение минимального числа точек считывания информации заключается в покрытии исходного множества точек (узлов) сети минимальным числом кругов радиуса $R$. Для решения такой задачи предлагается использовать итерационный метод кластерного анализа, в частности метод FOREL (FORmal ELement) [2, 11].

\section{Алгоритм реализации метода FOREL}

Алгоритм FOREL решает задачу кластеризации, при решении которой минимизируется суммарное квадратичное расстояние элементов кластеров (узлов БСС) от центров масс этих кластеров. Минимизируемая функция может быть записана как:

$$
M=\sum_{i=1}^{k} \sum_{x_{j} \in S_{i}}\left(x_{j}-\mu_{i}\right)^{2},
$$

где $k$ - число кластеров; $S_{i}$ - множество элементов $i$-го кластера; $\mu_{i}-$ координаты центра масс $i$-го кластера; $x_{j}$ - координаты центра масс $j$-го элемента кластера. 
Значение $\left(x_{j}-\mu_{i}\right)$ представляет собой эвклидово расстояние между элементом кластера и центром масс кластера. Рассмотрим данный алгоритм для двумерного пространства (плоскости). В данном случае каждый элемент рассматривается как точка на плоскости и характеризуется своими координатами $\left(x_{j}, y_{j}\right)$. Координаты центра масс $i$-го кластера определяются как:

$$
x_{i}^{\mu}=\frac{1}{n_{i}} \sum_{i=1}^{n_{i}} x_{j} y_{i}^{\mu}=\frac{1}{n_{i}} \sum_{i=1}^{n_{i}} y_{j} .
$$

Также каждый объект (точка) может характеризоваться кроме координат некоторым параметром («массой») $m_{j}$. Тогда центр масс будет определяться как (центр масс плоской фигуры):

$$
x_{i}^{\mu}=\frac{1}{m_{i}^{\Sigma}} \sum_{j=1}^{n_{i}} m_{j} x_{j}, \quad y_{i}^{\mu}=\frac{1}{m_{i}^{\Sigma}} \sum_{j=1}^{n_{i}} m_{j} y_{j}, \quad m_{i}^{\Sigma}=\sum_{j \in S_{i}} m_{j} .
$$

В алгоритме FOREL задается размер кластера. В двумерной задаче на геометрической плоскости под $R$ понимается максимальное расстояние от элемента кластера до его центра масс (радиус). Каждый элемент также рассматривается как точка на плоскости и характеризуется своими координатами $\left(x_{j}, y_{j}\right)$. Координаты центра масс $i$-го кластера определяются согласно (2) или (3). Алгоритм кластеризации включает в себя следующие основные шаги:

1. Задаются границы области, координаты объектов (точек) $I=\left\{I_{1}, I_{2}, \ldots, I_{n}\right\}$ и максимальный размер кластера $(R)$, номер кластера.

2. Выбирается случайная точка $m_{i}$ в заданной области. Эту точку на начальном этапе принимаем за центр масс кластера.

3. Все объекты, находящиеся на расстоянии не более $R$, приписываются к данному кластеру.

4. Для полученного кластера вычисляется центр масс $m_{i}$, согласно формулам (2) или (3). Если вычисленные координаты центра масс совпадают с точкой $m_{i}$, то полагаем, что кластер $i$ определен, все приписанные данному кластеру точки помечаются номером кластера и исключаются из дальнейшего рассмотрения. Переход к п. 5 (поиск следующего кластера). Если вычисленные координаты центра масс не совпадают с точкой, то процесс поиска продолжается, т. е. принимаем $m_{i}=m_{i}^{\prime}$, переход к п. 3 .

5. Проверяем, остались ли объекты, не отнесенные ни к одному кластеру. Если нет, то все кластеры определены, завершение поиска (к п. 6). Если объекты остались, то переход к п. 2 (поиск очередного кластера). 
Міжвідомчий науково-технічний збірник «Адаптивні системи автоматичного управління» № 2' (33) 2018

6. Выводим данные о принадлежности объектов к кластерам, центре масс полученных кластеров. Конец поиска.

Примечание. Во время поиска очередного кластера может оказаться, что на удалении менее от выбранного центра масс нет ни одного объекта. В таком случае следует произвести выбор нового центра масс (случайный выбор). Обобщенная схема-алгоритма FOREL приведена на рисунке 2.

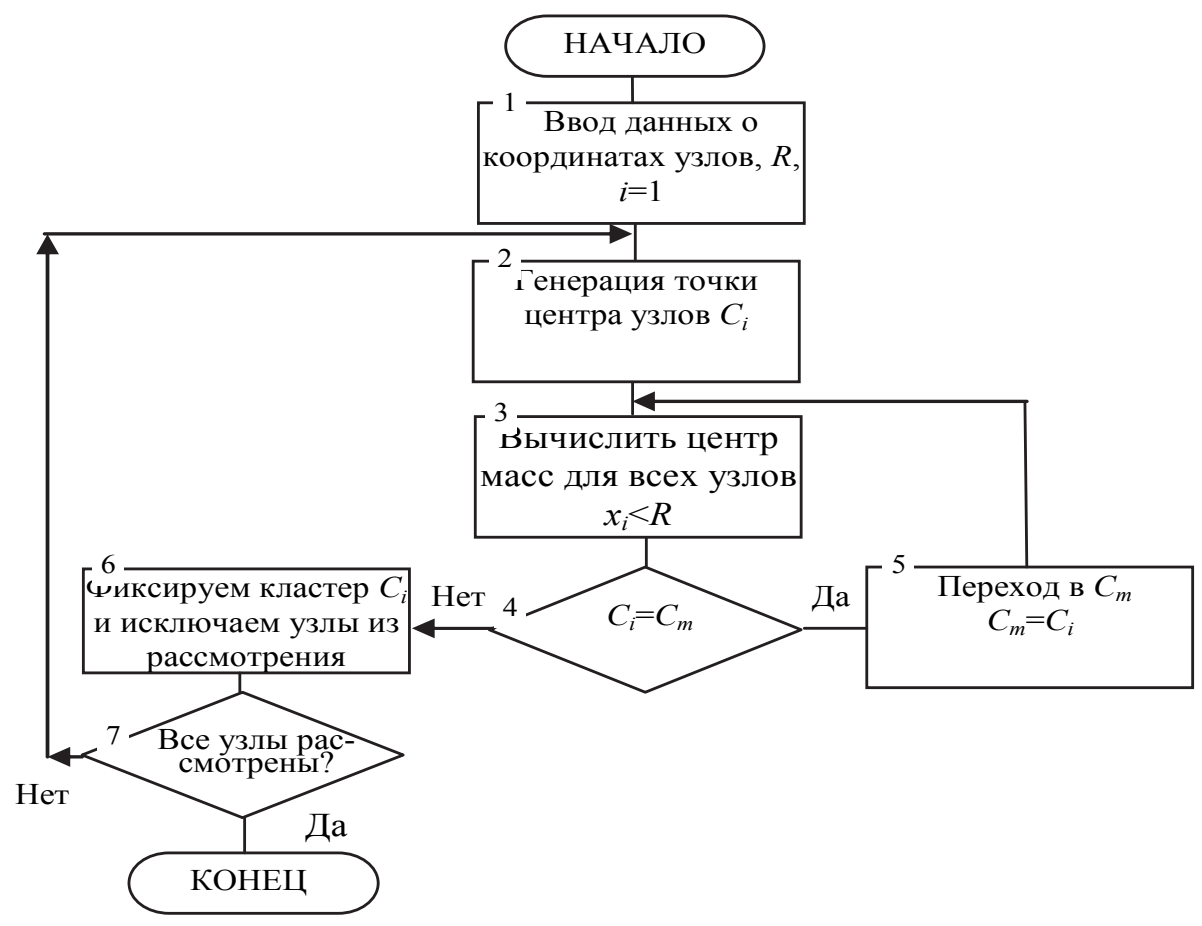

Puc. 2. Схема-алгоритм FOREL

Варианты решения для конкретного примера БСС представлены на рис. 3.

К достоинствам данного алгоритма можно отнести: незначительную вычислительную сложность $O\left(n^{2}\right)$, возможность изменения числа кластеров в зависимости от величины $R$, сходимость алгоритма увеличивается с увеличением $R$. Недостаток: конечное решение сильно зависит от начального приближения.
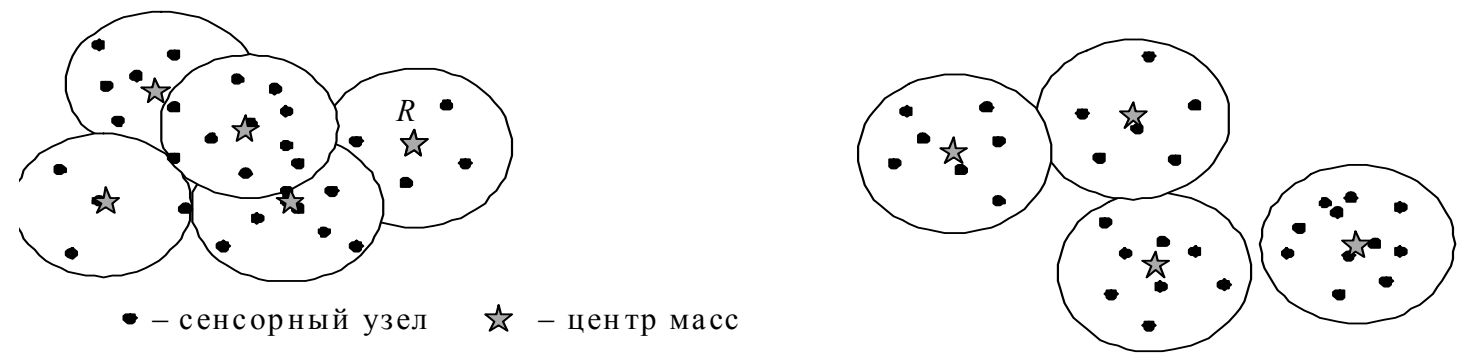

Puc. 3. Варианты решения при различном размещении сенсорных узлов 


\section{Решение задачи}

В отличие от ранее предложенных подходов решения задачи непосредственного сбора данных БпЛА от узлов БСС будем учитывать ряд целевых функций управления сетью:

1. Минимизащчия (ограничение) времени сбора $T_{\text {со }}$ информацчии мониторинга - за минимальное (ограниченное) время полета (один круг облета) собрать данные мониторинга от всех (выделенных) сенсорных узлов

$$
T_{c б} \rightarrow \min \text { или } T_{c б} \leq T_{\text {сбзад }}
$$

при ограничениях на количество БПЛА $N_{\text {БпЛА }} \leq N_{\text {БпЛАзад }}$, заданный объем дан-

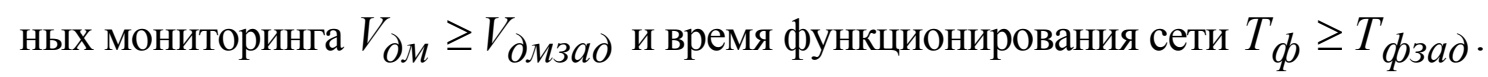

Время сбора данных зависит как от количества БпЛА, их технических характеристик (скорость, высота, дальность, продолжительность полета, радиосредств и др.), условий полета (характер местности, погодные условия и т.д.), так и от траектории движения.

Траектория движения БПЛА зависит от координат размещения узлов сети на территории. Если дальность радиосвязности БПЛА позволяет обеспечить обмен данными с некоторым количеством узлов БСС, то целесообразно найти точки сбора, в которых может быть обслужено максимальное количество узлов. Тогда траектория движения БпЛА будет линией, соединяющей данные точки. От выбора траектории существенно зависит длина маршрута БПЛА, а, следовательно, и время сбора и доставки данных мониторинга. В тоже время от высоты полета и дальности радиосвязности, зависит затрачиваемая мощность на передачу и скорость (время) обмена. Поэтому время обмена данными не должно превышать время существования радиосвязности при полете БпЛА над узлом. В табл. 1 представлены зависимости скорости передачи от дальности при использовании протокола IEEE 802.11g [5].

Увеличение дальности радиосвязности приводит к увеличению расхода энергии на передачу. Кроме этого предельное увеличение дальности радиосвязности ограничено малой мощностью передатчиков узлов. Пример различных значения зон радиосвязности $R$ от высоты полета $h$ БпЛА при дальности передачи $r_{n}=250 м$ (БПЛА та сенсорных узлов) показаны на рис. 4 [11].

2. Максимизация времени функиионирования сенсорной сети $T_{\phi}$.

$$
\begin{aligned}
& T_{\phi} \rightarrow \max \text { или } T_{\phi} \geq T_{\text {фзад }}, \\
& T_{c б} \leq T_{\text {сбзад }}, V_{\partial м} \geq V_{\text {дмзад }}, N_{\text {БпЛА }} \leq N_{\text {БпЛАзад }},
\end{aligned}
$$

при ограничениях на заданные: время $T_{c \sigma}$, объемы $V_{\partial м}$ сбора данных мониторинга (ДМ) и количество БПЛА $N_{\text {БпЛА }}$. 
Таблица 1. Зависимость скорости передачи от расстояния для стандарта IEEE $802.11 \mathrm{~g}$ при $B E R \leq 10^{-5}$

\begin{tabular}{|c|c|c|c|}
\hline $\begin{array}{c}\text { Скорость передачи } \boldsymbol{s}, \\
\text { Мб/с }\end{array}$ & Расстояние, $\mathbf{\text { м }}$ & Скорость передачи $\boldsymbol{s , ~ М б / c ~}$ & Расстояние, $\mathbf{M}$ \\
\hline \multicolumn{3}{|c|}{ Для коэффициента затухания $\alpha=2.25$ (между узлом и БпЛА) } \\
\hline 54 & 54 & 12 & 204 \\
38 & 69 & 9 & 219 \\
24 & 114 & 6 & 261 \\
18 & 146 & 2 & 328 \\
\hline \multicolumn{4}{|c|}{ Для коэффициента затухания $\alpha=3.0$ (между узлами БСС) } \\
\hline 48 & 20 & 12 & $d_{\max }$ \\
36 & 24 & 9 & 54 \\
24 & 35 & 2 & 65 \\
18 & 42 & 1 & 77 \\
\hline
\end{tabular}
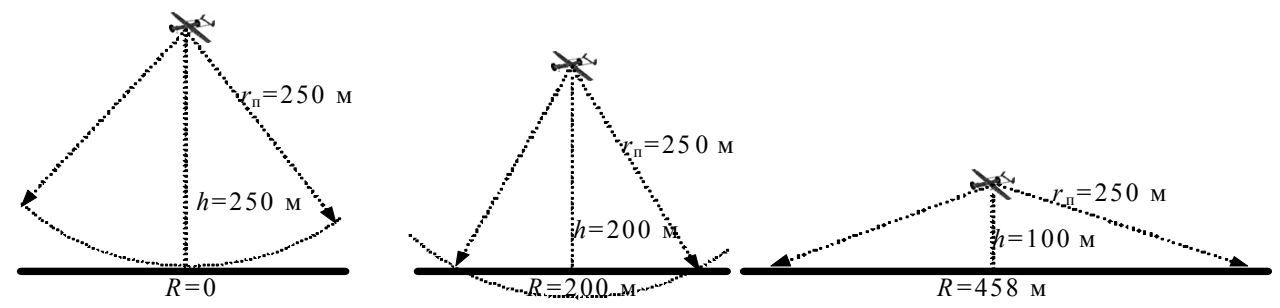

Puc. 4. Зависимости размера зоны радиосвязности $R$ от высоты полета при $r_{n}=250 \mu$.

Понятие “время функционирования” $T_{\phi}$ может трактоваться различными способами и будет определяться пользователем сети [10]:

- время работы сети до выхода из строя первого узла из-за израсходования энергии его батареи (так называемый период стабильной работы сети);

- время работы сети до выхода из строя $k$-критических узлов сети, определяющих функционал сети;

- время работы сети до выхода из строя части или всех узлов.

Увеличения времени функционирования сети будем добиваться применением специальных правил уменьшения расхода энергии батарей узлов при передаче за счет уменьшения расстояния между сенсорным узлом и БпЛА:

1. Минимальная высота полета БпЛА для конкретного кластера (подбор величины зоны радиосвязности $R$ ).

2. Назначение точек обмена данными для узлов, находящихся ближайших к траектории полета (рис. 5a).

3. Облет узлов с малой оставшейся энергией батарей на предельно малом расстоянии. 
4. Кооперативная работа группы близко расположенных узлов (формирование миникластера и реализация способа рандеву): выбор узла-рандеву, ближайшего к маршруту полета БпЛА, построение кратчайших маршрутов передачи от соседних узлов к узлу-рандеву с метриками: минимальная мощность передачи и оставшаяся энергия батарей (рис. 5б). Так, например, мощность, затрачиваемая

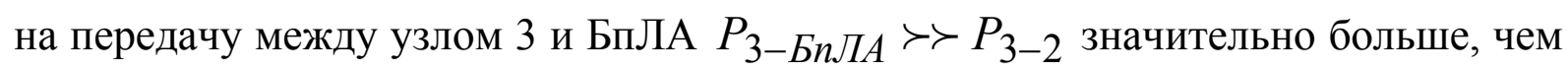
мощность, затрачиваемая на передачу между 3-м и 2-м узлами. И в целом для данного фрагмента сети

$$
\left(P_{3-2}+P_{2-1}+P_{1-\text { БпЛА }}\right) \prec\left(P_{1-\text { БnЛА }}+P_{2-\text { БпЛА }}+P_{3-\text { БпЛА }}\right) .
$$

Метрика выбора маршрута будет определяться соотношением $w_{i j}=P_{i j} \cdot l / s$, где $P_{i j}$ - мощность передачи на передачу при обеспечении заданного соотношения сигнал-шум, $l$ - длина пакета данных, $s$ - скорость передачи данных. Применив алгоритм поиска кратчайшего пути (например, Беллмана-Форда) с данной метрикой, мы можем найти оптимальные пути передачи между узлами миникластера и БпЛА.

5. Установка мощности передачи на минимально необходимом уровне.

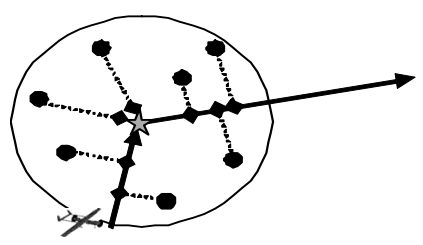

a)

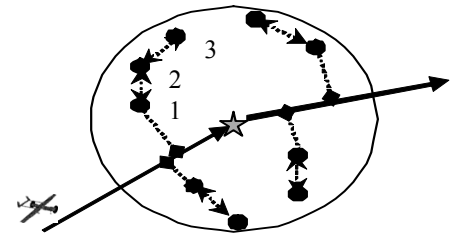

б)

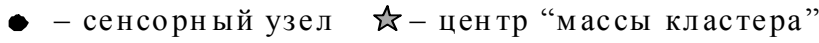

— - траектория полета БпЛА - точка сбора данных от узлак БпЛА

Puc. 5. Правила уменьшения расхода энергии при передаче данных на БпЛА

3. Минимизаџия количества БпЛА, необходимых для сбора данных с заданнымм качеством

$$
N_{\text {БпЛА }} \rightarrow \min ,
$$

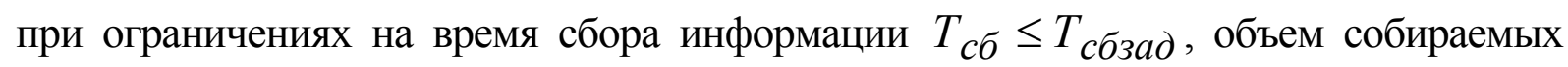

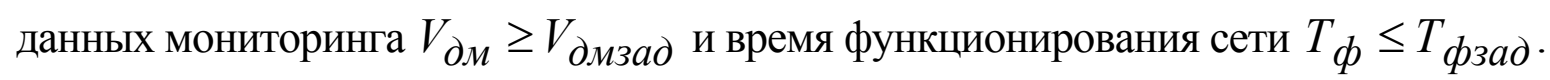

\section{Обобщенный алгоритм сбора данных}

1. Ввод исходных данных: множество узлов $i=1 \ldots N$ и их координаты размещения $\left(x_{i}, y_{i}\right)$; множество БПЛА $b=1 \ldots B$; исходная и конечная точка полета 
Міжвідомчий науково-технічний збірник «Адаптивні системи автоматичного управління» № 2' (33) 2018

БПЛА; максимальная и средняя v скорости, дальность полета; высота полета, дальности радиосвязности БПЛА и узлов, определяющие значение радиуса покрытия $R=\left[R_{\min }, R_{\max }\right]$ (начальное значение $R=R_{\max }$ ); заданное время сбора

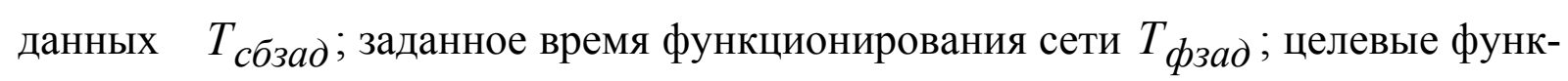
ции управления (4)-(6) и иерархия их приоритетов.

2. Нахождение количества и координат точек сбора данных мониторинга $\left(x_{b}, y_{b}\right)$ при помощи алгоритма FOREL при заданном значении $R$.

3. Построение базового маршрута полета БпЛА по полученным точкам сбора одним из известных методов поиска кратчайшего маршрута (задача коммивояжёра).

4. ЕСЛИ приоритет целевой функции $\min T_{c \sigma}$

ТО разделить маршрут облета на все БпЛА и переход к шагу 7.

5. Вычисление времени сбора данных БпЛА:

$T_{c \sigma}=L_{\mathcal{M}} / v$ - соотношение длины маршрута облета $L_{\mathcal{M}}$ к средней скорости полета $v$.

6. Проверка выполнения заданных требований по времени сбора данных:

ЕСЛИ $T_{c \sigma} \geq T_{\text {сбзад }}$ ТО

ЕСЛИ $\{b\} \neq \varnothing$ (проверка наличия резерва БпЛА)

ТО добавить БПЛА, разбить граф сети на части и выполнить алгоритм для каждого подграфа отдельно, переход к шагу 2.

ИНАЧЕ КОНЕЦ;

ИНАЧЕ $R^{\prime}=k R$ - уменьшить значение $R$ на определенную величину $k$;

ЕСЛИ $R^{\prime} \prec R^{\prime} \min$ ТО переход к шагу 7.

ИНАЧЕ переход к шагу 2 с новым $R=R$,

7. Реализация правила 3 - скорректировать базовый маршрут для облета на ближайшем расстоянии узлов с критичной энергией батарей (при выполнении условия $\left.T_{c б} \leq T_{\text {сбзад}}\right)$.

8. Реализация правила 2 - назначить точки обмена данными, ближайшие к траектории полета БпЛА (рис. 5а).

9. Реализация правила 4 - организовать кооперативную работу близкорасположенных узлов (миникластера) по построению энергоэффективных маршрутов передачи данных к узлу-рандеву, ближайшему к траектории полета БпЛА (рис. 5б). КОНЕЦ.

Так как в исходных данных возможно упорядочивание критериев по важности, то задачу многокритериальной оптимизации будем решать, используя 
метод последовательных уступок. В случае приоритета $\min T_{c \sigma}$ шаг 4 алгоритма распределяет маршрут облета между всеми БпЛА. Начальная реализация алгоритма со значением $R=R_{\max }$ пытается минимизировать количество точек сбора и соответственно время сбора данных. Затем при удовлетворении

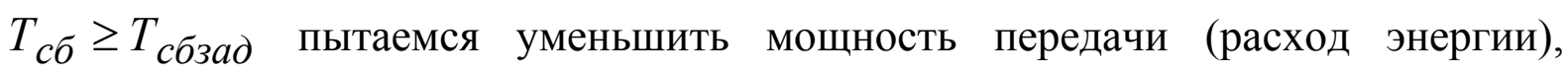
уменьшая $R$ и применяя соответствующие правила уменьшения мощности передачи. Реализация правил возможна только при наличии соответствующих алгоритмов управления в узлах сети. При удовлетворении требований (ограничений) по времени сбора, величине расходуемой энергии узлов алгоритм стремится минимизировать число задействованных БпЛА, добавляя при каждой итерации решения только по одному БпЛА.

Для практической проверки результатов функционирования алгоритма осуществлена его программная реализация в среде MATLAB. Проведены эксперименты и получены зависимости:

- времени сбора данных и времени стабильного функционирования сети от размерности сети; среднего расхода энергии узлов при различном количестве облетов при применении различных алгоритмов непосредственного сбора данных. Показано, что предложенный алгоритм позволяет в среднем на 15-20\% сократить время облета и на 10-15\% увеличить время стабильного функционирования сети в сравнении и известными алгоритмами непосредственного сбора данных.

Выводы. Таким образом, для временной кластеризации беспроводной сенсорной сети предложено использовать БпЛА, который реализует модифицированный итерационный алгоритма кластерного анализа FOREL. Предложенный алгоритм сбора данных позволяет:

- сократить маршрут облета и, соответственно, время сбора данных за счет минимизации количества кластеров (точек сбора данных мониторинга);

- увеличить время функционирования сети за счет уменьшения мощностей (дальности) передач между БпЛА и сенсорными узлами, адаптации радиуса покрытия БПЛА к реальному размещению узлов кластера, нахождения ближайших точек обмена данными от маршрута перемещения БпЛА, построения энергоэффективных маршрутов передачи данных между узлами сети и БпЛА;

- уменьшить количество используемых БПЛА.

Направлением дальнейших исследований является оценка эффективности предложенного алгоритма при различных протоколах обмена (случайного и детерминированного множественного доступа) между БпЛА и сенсорными узлами в кластерах. 


\section{Список використаних джерел}

1. Imad Jawhar, Nader Mohamed, Jameela Al-Jarood UAV-based data communication in wireless sensor networks: Models and Strategies, International Conference on Unmanned Aircraft Systems (ICUAS), 2015.

2. Киричек Р.В. Разработка и исследование комплекса моделей и методов для летающих сенсорных сетей: Диссертация на соискание ученой степени доктора технических наук: 05.12.13 / Киричек Руслан Викторович. - СПб, 2017. - 316 с.

3. Dac-Tu Ho, Esten Ingar Grøtli, P. B. Sujit, Tor Arne Johansen, João Borges Sousa Optimization of Wireless Sensor Network and UAV Data Acquisition Journal of Intelligent \& Robotic Systems April 2015, Volume 78, Issue 1, pp 159 - 179.

4. Huseyin Okcu, Mujdat Soyturk. Distributed Clustering Approach for UAV Integrated Wireless Sensor Networks, International Journal of Ad Hoc and Ubiquitous Computing, 2014, Vol.15, No. 1-3, pp.106 - 120.

5. Dac-Tu Ho, Esten Ingar Grotli, and Tor Arne Johansen Heuristic Algorithm and Cooperative Relay for Energy Efficient Data Collection with a UAV and WSN. http://folk.ntnu.no/torarnj/ho_2013.pdf.

6. Гурник А.В. Застосування інтелектуальної сенсорної техніки для моніторингу та пошуко-рятувальних робіт / А.В. Гурник, С.В. Валуйский // Восточноевропейский журнал передовых технологий. - 2013. - № 3 (9). - 27 - 32.

7. Романюк А.В. Задачи управління збором даних мониторингу БпЛА в беспроводових сенсорных мережах / А.В. Романюк // Збірник наукових праць ВITI. - 2018. № 2. - C. $103-112$.

8. Романюк А.В. Метод сбора информации мониторинга в беспроводных сенсорных сетях с использованием БпЛА / А.В. Романюк // Збірник наукових праць ВІТІ. 2018. - № 1. - C. 90 - 99.

9. Sarmad Rashed and Mujdat Soyturk Analyzing the Effects of UAV Mobility Patterns on Data Collection in Wireless Sensor Networks, Sensors 2017, 17, 413; doi:10.3390/s17020413.

10. Романюк А.В. Моделі оцінки ефективності функціонування беспроводових сенсорних мереж / А.В. Романюк // Збірник наукових праць ВITI. - 2017. - № 3. C. $125-134$.

11. Загоруйко Н. Г. Прикладные методы анализа данных и знаний. - Новосибирск: ИМ СО РАН, 1999. - 270 с. 